\title{
Neues vom neuen Lohnausweis
}

\section{P. Salathe}

Das Thema «Neuer Lohnausweis» beschäftigt und erhitzt die Gemüter seit einiger Zeit. Betroffen von der Einführung des neuen Lohnausweises sind nicht nur die steuerpflichtigen Lohnausweisempfänger, sondern genauso deren Arbeitgeber, welche diese Deklarationen zu erstellen haben. Wirtschaftsverbände laufen Sturm gegen die Einführung dieses vieldiskutierten und ebenso umstrittenen Formulars. Die «Risiken und Nebenwirkungen» des neuen Lohnausweises für Arbeitgeber und Arbeitnehmer sind nämlich immer noch nicht absehbar, dazu fehlt schlicht und einfach die Praxis, welche sich verständlicherweise erst nach Einführung zeigen wird.

\section{Worum geht es?}

Der Lohnausweis ist die wichtigste Grundlage für die Besteuerung des Arbeitnehmers und für Abzugsmöglichkeiten bei den Berufsauslagen. Das heute noch geltende Lohnausweisformular ist rund dreissig Jahre alt und es existieren nebst diesem noch verschiedene kantonale Versionen. Mit der Einführung des Steuerharmonisierungsgesetzes hat die Schweizerische Steuerkonferenz (SSK) beschlossen, ein neues, gesamtschweizerisch einheitliches Lohnausweisformular zu erstellen und neue Vorschriften dazu zu erlassen. Dieser neue Lohnausweis wurde 2001 in die Vernehmlassung geschickt. Seit dann wurden ihm viele Zähne gezogen und die Einführung wurde mehrfach verschoben. Auf das Jahr 2007 hin soll er nun definitiv eingeführt werden. Gemäss SSK wurde damit die Einführung ein letztes Mal verschoben. Im Rahmen des Pilotprojektes darf dieser freiwillig schon für Löhne ab 2005 und 2006 eingesetzt werden.

\section{Alter Wein in neuen Schläuchen?}

Der neue Lohnausweis und die zugehörige Wegleitung sollen vor allem Transparenz und Klarheit schaffen. Die Steuerverwaltung möchte Auskünfte bezüglich sämtlicher Lohnbezüge. Nebst dem Gehalt verlangt die Steuerverwaltung auch detaillierte Auskünfte zu allen Lohnnebenleistungen oder auf neudeutsch «Fringe Benefits». Diese sind vielfältig und hätten eigentlich schon mit dem geltenden Lohnausweisformular und nach geltender Steuerpraxis der Besteuerung zugeführt werden sollen. Jedoch wurden die Regelungen zum alten Lohnausweis bisher weder von den Arbeitgebenden noch von den Verwaltungen so konsequent umgesetzt. Nach Schätzungen von Experten ist nämlich eine Mehrheit der heute ausgestellten Lohnausweise falsch ausgefüllt. Dies birgt grosse Gefahren vor allem für den ausstellenden Arbeitgeber, denn der Lohnausweis hat Urkundencharakter, und eine Falschdeklaration kann daher eine Urkundenfälschung sein!

Mit dem neuen Lohnausweis wird auch die 18seitige «Wegleitung zum Ausfüllen des Lohnausweises bzw. der Rentenbescheinigung» (www.steuerkonferenz.ch oder www.estv.admin. ch) in Kraft treten. Diese gibt detailliert Auskunft darüber, was wo in welcher Höhe deklariert werden muss. So kommen auf das neue Formular neben dem ordentlichen Salär auch sämtliche Zulagen (Kinder- bzw. Familienzulagen, Schicht-, Pikett-, Versetzungs-, Nacht-, Sonntags-, Schmutzund Wegzulagen, Prämien), Provisionen, Vergütungen für den Arbeitsweg und alle Barbeiträge für die auswärtige Verpflegung am Arbeitsort. Sämtliche Gehaltsnebenleistungen sind zum Markt- bzw. Verkehrswert ebenso zu deklarieren wie vom Arbeitgeber zur Verfügung gestellte Verpflegung und Unterkunft (Zimmer), Privatanteile an Geschäftswagen und andere Gehaltsnebenleistungen, Kapitalleistungen und Leistungen aus Beteiligungsrechten, Verwaltungsentschädigungen und Spesen verschiedenster Art.

Die Regelungen zu den einzelnen zu deklarierenden Beträgen werden in der Wegleitung ausführlich beschrieben. Hier nur ein Beispiel: Für die Ermittlung des Privatanteils an Geschäftswagen steht eine Pauschalvariante (pro Monat 1\% des Kaufpreises) zur Verfügung. Bei speziellen Gegebenheiten kann vom Sitzkanton ein angepasster Privatanteil von weniger als 1\% pro Monat bewilligt werden. Diese Tatsache wird dann mit folgendem Vermerk kundgetan: «Privatanteil für Geschäftswagen durch Kanton $\mathrm{X}$ (Autokennzeichen des Kantons) am ... (Datum) genehmigt». Wenn der Arbeitnehmer beträchtliche Kosten selbst trägt, dann ist der Text «Privatanteil im Veranlagungsverfahren abzuklären» anzubringen. Es geht aber auch noch komplizierter: Der Privatanteil kann auch durch ein über das ganze Jahr hindurch zu führendes Bordbuch, in welchem sämtliche gefah- 
renen Kilometer eingetragen werden, effektiv ermittelt werden. Welche Variante man auch immer wählt, es muss dann auf jeden Fall noch das Feld $\mathrm{F}$ «unentgeltliche Beförderung zwischen Wohn- und Arbeitsort» angekreuzt werden.

\section{Risiken und Nebenwirkungen}

Aufgrund solcher Bestimmungen liegt der Schluss nahe, dass die neu geschaffene Klarheit und Transparenz nicht nur zu einem höheren Steuersubstrat führt, sondern dass den Arbeitgebern ein erheblicher administrativer Mehraufwand aufgebürdet wird. Schliesslich müssen die neu zu deklarierenden Daten gesammelt, verwaltet und die Lohnsoftware angepasst werden. Zudem werfen gerade die detaillierteren Vorschriften wieder neue Fragen zur Auslegung aus. Und die Auslegung wird sich erst nach Einführung des Lohnausweises in der Praxis zeigen. Hier kommt es ganz darauf an, was die Verwaltung daraus macht. So hat sich die vor der Einführung als einfach angepriesene Mehrwertsteuer zu einer höchst komplexen und anspruchsvollen Steuer entwickelt.

Ein weiteres Risiko wird in der Tatsache befürchtet, dass allfällige jetzige unwissentliche Falschdeklarationen durch den neuen Lohnausweis aufgedeckt werden könnten. Die Steuerbehörden könnten im schlimmsten Falle gegen Mitarbeiter Nach- und Strafsteuerverfahren einleiten und die Arbeitgeber wegen Urkundenfälschung belangen.

\section{Wie geht es weiter?}

Gemäss SSK wird der neue Lohnausweis definitiv auf das Jahr 2007 eingeführt. Im Moment sind jedoch noch viele politische Vorstösse hängig. Ob diese Begehren die Einführung des neuen Lohnausweises weiter verzögern oder sogar verhindern werden, steht noch in den Sternen.

Den arbeitgebenden Ärzten und Ärztinnen wie auch Spitälern und anderen Gesundheitseinrichtungen kann zum heutigen Zeitpunkt nur empfohlen werden, sich mit dem neuen Lohnausweis auseinanderzusetzen und Anpassungen in der Software und in der Datenverwaltung so weit vorzubereiten, dass diese vor dem 1. Januar 2007 problemlos getestet und eingeführt werden können. Dazu gehört auch die Überprüfung bestehender Reglemente (Spesen-, Aus- und Weiterbildung) und allenfalls die Einführung eines von der Steuerverwaltung genehmigten Spesenreglementes. Zudem müssen Zahlungen und andere Leistungen, welche den Arbeitnehmenden bisher ohne Erfassung in der Lohnbuchhaltung zugeflossen sind, identifiziert und erfasst werden. Werden heute steuerrechtlich eher als heikel einzustufende Leistungen ausgerichtet, sollten diese frühzeitig entsprechend korrigiert werden. Um dies alles umsetzen zu können, bedarf es sicherlich auch der Schulung der mit dem Ausfüllen der Lohnausweise betrauten Personen und/oder des Zuzugs von Fachleuten. 
A Lohnausweis - Certificat de salaire - Certificato di salario

B Rentenbescheinigung - Attestation de rentes - Attestazione delle rendite

C AHV-Nr. - No AVS - N. AVS

D E Jahr - Année - Anno von - du - dal
$\mathbf{F}$

G
Unentgeltliche Beförderung zwischen Wohn- und Arbeitsort Transport gratuit entre le domicile et le lieu de travail Trasporto gratuito dal domicilio al luogo di lavoro

Kantinenverpflegung / Lunch-Checks Repas à la cantine / chèques-repas Pasti alla mensa / buoni pasto

H

1. Lohn soweit nicht unter Ziffer 2-7 aufzuführen / Rente

Salaire qui ne concerne pas les chiffres 2 à 7 ci-dessous / Rente

Salario se non da indicare sotto cifre da 2 a 7 più sotto / Rendita

2. Gehaltsnebenleistungen

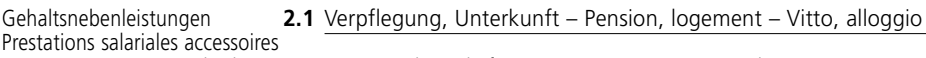

Prestazioni accessorie al salario 2.2 Privatanteil Geschäftswagen - Part privée voiture de service - Quota privata automobile di servizio 2.3 Andere - Autres - Altre Art - Genre - Genere

3. Unregelmässige Leistungen - Prestations non périodiques - Prestazioni aperiodiche

Art - Genre - Genere

4. Kapitalleistungen - Prestations en capital - Prestazioni in capitale Art - Genre - Genere

5. Beteiligungsrechte gemäss Beiblatt - Droits de participation selon annexe - Diritti di partecipazione secondo allegato

6. Verwaltungsratsentschädigungen - Indemnités des membres de l'administration - Indennità dei membri di consigli d'amministrazione

7. Andere Leistungen Art Autres prestations Genre Altre prestazioni Genere

8. Bruttolohn total / Rente - Salaire brut total / Rente - Salario lordo totale / Rendita

9. Beiträge AHV/IV/EO/ALV/NBUV - Cotisations AVS/AI/APG/AC/AANP - Contributi AVS/AI/IPG/AD/AINP

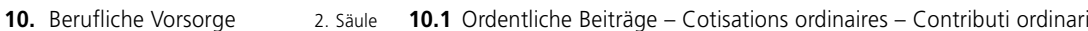
Prévoyance professionnelle 2e pilier Previdenza professionale $\quad 2^{\circ}$ pilastro 10.2 Beiträge für den Einkauf - Cotisations pour le rachat - Contributi per il riscatto

11. Nettolohn / Rente - Salaire net / Rente - Salario netto / Rendita In die Steuererklärung übertragen - A reporter sur la déclaration d'impôt - Da riportare nella dichiarazione d'imposta

12. Quellensteuerabzug - Retenue de l'impôt à la source - Ritenuta d'imposta alla fonte

13. Spesenvergütungen - Allocations pour frais - Indennità per spese Nicht im Bruttolohn (gemäss Ziffer 8) enthalten - Non comprises dans le salaire brut (au chiffre 8) - Non comprese nel salario lordo (sotto cifra 8) 13.1 Effektive Spesen 13.1.1 Reise, Verpflegung, Übernachtung - Voyage, repas, nuitées - Viaggio, vitto, alloggio Frais effectifs Frais effectifs

13.1.2 Übrige - Autres - Altre

3.2 Pauschalspesen Frais forfaitaire Spese forfettarie

13.2.1 Repräsentation - Représentation - Rappresentanza

13.2.2 Auto - Voiture - Automobile

$$
\text { 13.2.3 Übrige - Autres - Altre }
$$

13.3 Beiträge an die Weiterbildung - Contributions au perfectionnement - Contributi per il perfezionamento

14. Weitere Gehaltsnebenleistungen Art Autres prestations salariales accessoires Genre Altre prestazioni accessorie al salario Genere

15. Bemerkungen Observations Osservazioni

I Ort und Datum - Lieu et date - Luogo e data Die Richtigkeit und Vollständigkeit bestätigt inkl. genauer Anschrift und Telefonnummer des Arbeitgebers Certifié exact et complet

y. adresse et numéro de téléphone exacts de l'employeur

Certificato esatto e completo

Form. 11-2005-dfi (15.9.2004)

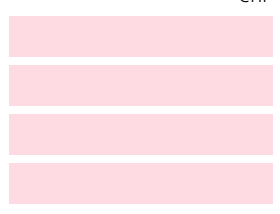

